\title{
Pulmonary endarterectomy in a toddler with chronic thromboembolic pulmonary hypertension after Denver shunt
}

\author{
Tilman Humpl, MD, PhD, ${ }^{\mathrm{a}}$ Osami Honjo, MD, PhD, ${ }^{\mathrm{b}}$ Michael Temple, $\mathrm{MD},{ }^{\mathrm{c}}$ and
} Marc de Perrot, MD, MSc, ${ }^{\mathrm{d}}$ Toronto, Ontario, Canada

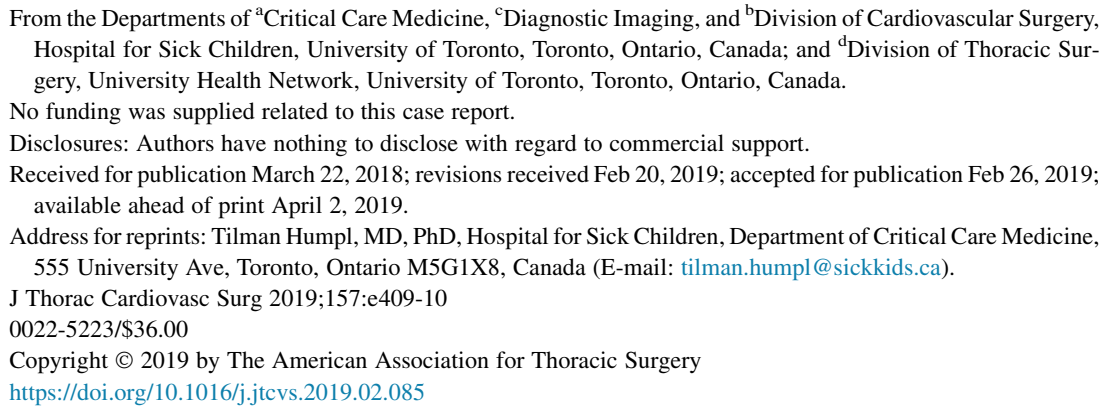

Chronic thromboembolic pulmonary hypertension (CTEPH) is a rare condition in the general population (incidence as great as 5.7 cases/million adults ${ }^{1}$ ). Although this is higher than previously reported, the diagnosis remains very infrequent in children. ${ }^{2}$ Ventriculoatrial and other artificial shunts to the central venous system are known risk factors for CTEPH. ${ }^{2}$ The Denver shunt, a peritoneovenous shunt first introduced in $1974,{ }^{3}$ is frequently used for chronic ascites in adults and children; however, to the best of our knowledge, its association with CTEPH has never been reported. Pulmonary thromboendarterectomy is the treatment of choice for CTEPH, other options include targeted medical therapy for pulmonary hypertension, and balloon pulmonary angioplasty. ${ }^{4}$

\section{CASE PRESENTATION}

We report the case of a toddler girl with intermediate-risk abdominal neuroblastoma diagnosed at 16 months of age. She was started on chemotherapy, with partial response, and underwent laparotomy with tumor resection at 21 months of age. She had significant ascites develop, and the decision was made to place a Denver shunt. As the ascites resolved, the shunt tip moved from the cavoatrial junction deeper into the atrium. At 2 years and 10 months, a routine echocardiogram revealed signs of pulmonary hypertension with moderate to severe right ventricular dilation and moderately reduced right ventricular systolic function, septal flattening and an estimated right ventricular systolic pressure of $68 \mathrm{~mm} \mathrm{Hg}$ (plus right atrial pressure), and mild tricuspid regurgitation.

As part of the workup, computed tomography showed bilateral pulmonary emboli with evidence of chronic thromboembolic disease and signs of pulmonary hypertension (Figure 1). The patient was started on a therapeutic dose

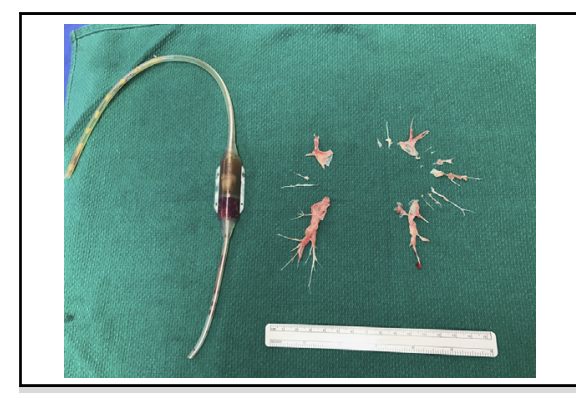

Denver shunt and cast of pulmonary endarterectomy.

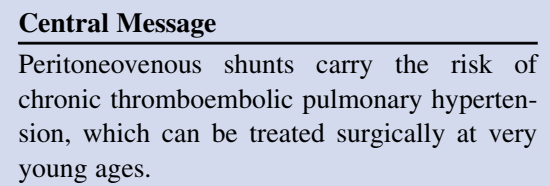

See Commentary on page e411. of low-molecular weight heparin and supplemental oxygen. Both echocardiography and computed tomography were repeated 6 weeks later and did not show any improvement.

A diagnosis of CTEPH was therefore established, and the decision was made to proceed to pulmonary thromboendarterectomy and removal of the Denver shunt. The procedure was performed through a sternotomy under cardiopulmonary bypass (162 minutes) with deep hypothermic circulatory arrest $\left(20^{\circ} \mathrm{C}\right)$. One circulatory arrest $(53$ minutes) was required for the right and the left sides (26 and $27 \mathrm{mi}-$ nutes, respectively). Needle measurement showed half systemic right ventricular pressure. The cast of endarterectomy was of type II according to the San Diego classification (Figure 2). Histologic examination showed evidence of organizing thrombus and pronounced fibrointimal thickening in a complicated arcardelike pattern without active infective process or evidence of a neoplasm.

The patient was sedated and ventilated in the cardiac critical care unit for 2 days and kept on standard heparin with therapeutic levels. She was extubated 2 days later and weaned off to room air by the fourth postoperative day. She eventually discharged from hospital 1 week after surgery on low-molecular weight heparin.

The echocardiogram revealed good biventricular systolic function, trivial tricuspid regurgitation (unable to estimate 


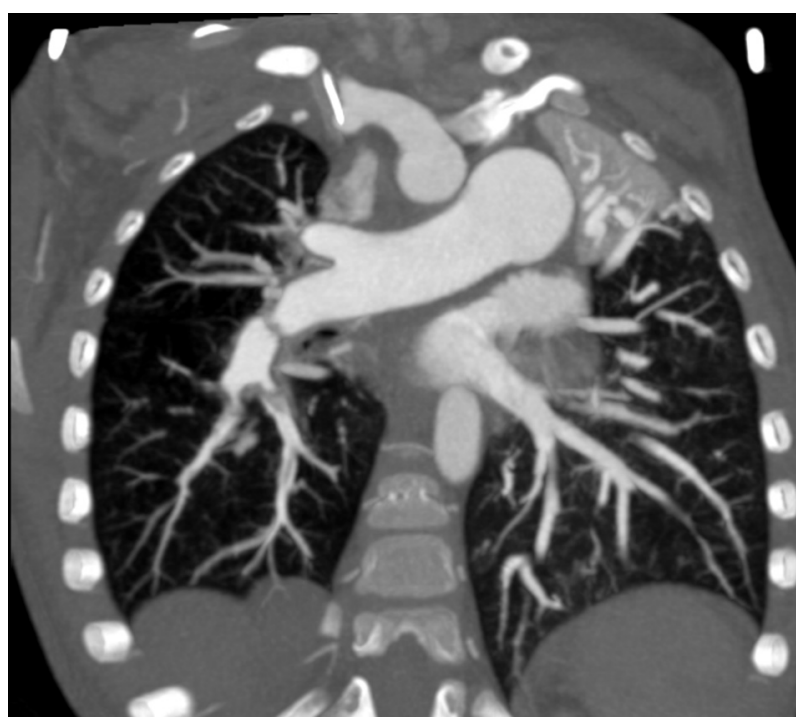

FIGURE 1. Computed tomography showing bilateral pulmonary emboli with evidence of chronic thromboembolic disease and signs of pulmonary hypertension.

right ventricular systolic pressure), and a normal septal curvature, demonstrating the resolution of the right ventricular pressure overload at 2 postoperative weeks and at 3 postoperative months. Low-molecular weight heparin was discontinued with normal echocardiographic findings 9 months after surgery.

\section{DISCUSSION}

In adults, short- and long-term survivals after surgical pulmonary endarterectomy are excellent, with a 10-year survival ranging between $80 \%$ and $90 \%$ in recent series. ${ }^{5}$ In children, the disease may be underdiagnosed. Data from a large registry showed that only $41 \%$ of patients with pulmonary hypertension diagnosed by cardiac catheterization underwent chest computed tomography and only $23 \%$ had a lung perfusion scan, highlighting the need of complete workup for children with elevated pulmonary pressures. ${ }^{6}$ To our knowledge, this patient is the youngest reported to have CTEPH diagnosed preoperatively and to then undergo successful bilateral pulmonary endarterectomy. A case series from San Diego on pediatric patients aged 8 to 17 years showed good success with major complications related to rethrombosis $(38 \%)$, moderate to severe pericardial effusion $(29 \%)$, and pulmonary arterial hypertension-targeted pharmacotherapy at discharge $(24 \%){ }^{2}$ In addition, a case report was published by the group in Paris of an infant with suspected acute pulmonary

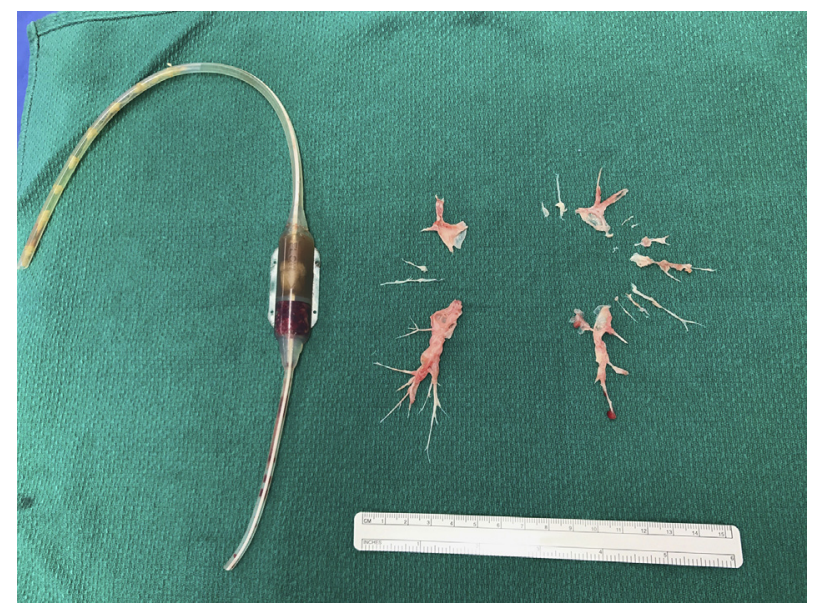

FIGURE 2. Denver shunt and cast of pulmonary endarterectomy.

emboli. $^{7}$ In our patient, the etiology is related to the Denver shunt, which was removed at the same time. We therefore elected not to start pulmonary arterial hypertension-targeted pharmacotherapy, because right sided pressures estimated by echocardiography normalized within 2 weeks and the clinical status dramatically improved after surgery, suggesting that there was no residual pulmonary hypertension. To avoid further radiation, we did not proceed with another computed tomographic scan. This report clearly emphasizes the potential complication of a peritoneoatrial shunt and the importance of seeking the cause of pulmonary hypertension, such as CTEPH, even at very young ages.

\section{References}

1. Kramm T, Wilkens H, Fuge J, Schäfers HJ, Guth S, Wiedenroth CB, et al. Incidence and characteristics of chronic thromboembolic pulmonary hypertension in Germany. Clin Res Cardiol. 2018;107:548-53.

2. Madani MM, Wittine LM, Auger WR, Fedullo PF, Kerr KM, Kim NH, et al. Chronic thromboembolic pulmonary hypertension in pediatric patients. J Thorac Cardiovasc Surg. 2011;141:624-30.

3. LeVeen HH, Christoudias G, Ip M, Luft R, Falk G, Grosberg S. Peritoneo-venous shunting for ascites. Ann Surg. 1974;180:580-91.

4. Yandrapalli S, Tariq S, Kumar J, Aronow WS, Malekan R, Frishman WH, et al. Chronic thromboembolic pulmonary hypertension: epidemiology, diagnosis, and management. Cardiol Rev. 2018;26:62-72.

5. Cannon JE, Pepke-Zaba J. Contemporary diagnosis and management of pulmonary hypertension. Heart. 2016;102:1680-91.

6. Beghetti M, Berger RM, Schulze-Neick I, Day RW, Pulido T, Feinstein J, et al. Diagnostic evaluation of paediatric pulmonary hypertension in current clinical practice. Eur Respir J. 2013;42:689-700.

7. Lambert V, Durand P, Devictor D, Planché C, Serraf A. Unilateral right pulmonary thromboendarterectomy for chronic embolism: a successful procedure in an infant. J Thorac Cardiovasc Surg. 1999;118:953-4; discussion 957. 